\title{
Inclusive semileptonic charmless B-decays in SUSY
}

\author{
Enrico Lunghi * \\ SISSA-ISAS, Via Beirut 2-4, Trieste, Italy and INFN, Sezione di Trieste, Trieste, Italy \\ E-mail: 'Iunghi@sissa.it'
}

Abstract: We study the semileptonic decays $B \rightarrow X_{s} e^{+} e^{-}, B \rightarrow X_{s} \mu^{+} \mu^{-}$in generic supersymmetric extensions of the Standard Model. SUSY effects are parameterized using the mass insertion approximation formalism and differences with MSSM results are pointed out. Constraints on SUSY contributions coming from other processes $(e . g . b \rightarrow s \gamma)$ are taken into account. Chargino and gluino contributions to photon and Z-mediated decays are computed and non-perturbative corrections are considered. We find that the integrated branching ratios and the asymmetries can be strongly modified. Moreover, the behavior of the differential Forward-Backward asymmetry remarkably changes with respect to the Standard Model expectation.

\section{KEYwords: 'rare semileptonic Beauty decays SUSYYY!}

\section{Introduction}

One of the features of a general low energy supersymmetric (SUSY) extension of the Standard Model (SM) is the presence of a huge number of new parameters. FCNC and CP violating phenomena constrain strongly a big part of the new parameter space. However there is still room for significant departures from the SM expectations in this interesting class of physical processes. It is interesting to check all these possibilities on the available data and on those processes that are going to be studied in the next future. In this way it is possible to indicate where new physics effects can be revealed as well as to establish criteria for model building.

In this work we want to investigate the relevance of new physics effects in the semileptonic inclusive decay $B \rightarrow X_{s} l^{+} l^{-}$. This decay is quite suppressed in the Standard Model; however, new $B$-factories should reach the precision requested by the SM prediction [i and an estimate of all possible new contributions to this process is compelling.

Because of the presence of so many unknown parameters (in particular in the scalar mass ma-

\footnotetext{
*Co-authors: Antonio Masiero, Ignazio Scimemi and Luca Silvestrini.
}

trices) which enter in a quite complicated way in the determination of the mass eigenstates and of the various mixing matrices it is very useful to adopt the so-called "Mass Insertion Approximation" (MIA) [i] chooses a basis for fermion and sfermion states in which all the couplings of these particles to neutral gauginos are flavor diagonal. Flavor changes in the squark sector are provided by the nondiagonality of the sfermion propagators. The pattern of flavor change is then given by the ratios

$$
\left(\delta_{i j}^{f}\right)_{A B}=\frac{\left(m_{i j}^{\tilde{f}}\right)_{A B}^{2}}{M_{s q}^{2}}
$$

where $\left(m_{i j}^{\tilde{f}}\right)_{A B}^{2}$ are the off-diagonal elements of the $\tilde{f}=\tilde{u}, \tilde{d}$ mass squared matrix that mixes flavor $i, j$ for both left- and right-handed scalars ( $A, B=$ Left, Right), $M_{s q}$ is the average squark mass (see e.g. $\left.\cdot{ }_{0}^{-1}\right)$. The sfermion propagators are expanded in terms of the $\delta$ s and the contribution of the first two terms of this expansion are considered. The genuine SUSY contributions to the Wilson coefficients will be simply proportional to the various $\delta$ s and a keen analysis of the different Feynman diagrams involved will allow us to isolate the few insertions really relevant for a given process. In this way we see that only a small 
number of the new parameters is involved and a general SUSY analysis is made possible. The hypothesis regarding the smallness of the $\delta$ s and so the reliability of the approximation can then be checked a posteriori.

We consider all the possible contributions to charmless semileptonic $B$ decays coming from chargino-quark-squark and gluino-quark-squark interactions and we analyze both Z-boson and photon mediated decays. Contributions coming from penguin and box diagrams are taken into account; moreover, corrections to the MIA results due to a light $\tilde{t}_{R}$ are considered. A direct comparison between the SUSY and the SM contributions to the Wilson coefficients is performed. Once the constraints on mass insertions are established, we find that in generic SUSY models there si still enough room in order to see large deviations from the SM expectations for branching ratios and asymmetries. For our final computation of physical observables we consider NLO order QCD evolution of the coefficients and nonperturbative corrections $\left(O\left(1 / m_{b}^{2}\right), O\left(1 / m_{c}^{2}\right), ..\right)$, each in its proper range of the dilepton invariant mass.

\section{General framework}

The effective Hamiltonian for the decay $B \rightarrow$

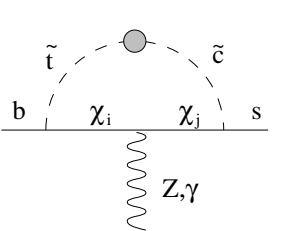

A

b

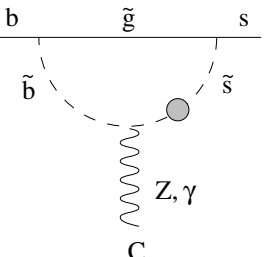

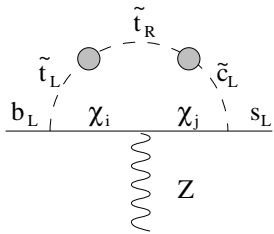

B

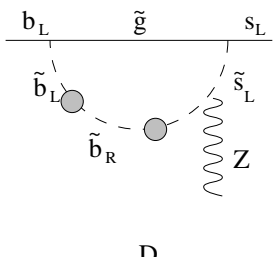

Figure 1: Some of the relevant Feynman diagrams for $b \rightarrow s l^{+} l^{-}$. Bubbles indicate Mass Insertions. Diagrams $A, B$ are based on chargino interaction. Diagrams $C, D$ consider gluino interactions.
$X_{s} l^{+} l^{-}$in the SM and in the MSSM is given by (neglecting the small contribution proportional to $\left.K_{u s}^{*} K_{u b}\right)$

$$
\mathcal{H}_{\mathrm{eff}}=-\frac{4 G_{F}}{\sqrt{2}} K_{t s}^{*} K_{t b} \sum_{i=1}^{10} C_{i}(\mu) Q_{i}
$$

where

$$
\begin{aligned}
Q_{1} & =\bar{s}_{L \alpha} \gamma_{\mu} b_{L \alpha} \bar{c}_{L \beta} \gamma^{\mu} c_{L \beta}, \\
Q_{2} & =\bar{s}_{L \alpha} \gamma_{\mu} b_{L \beta} \bar{c}_{L \beta} \gamma^{\mu} c_{L \alpha}, \\
Q_{3} & =\bar{s}_{L \alpha} \gamma_{\mu} b_{L \alpha} \sum_{q=u, ., b} \bar{q}_{L \beta} \gamma^{\mu} q_{L \beta}, \\
Q_{4} & =\bar{s}_{L \alpha} \gamma_{\mu} b_{L \beta} \sum_{q=u, . ., b} \bar{q}_{L \beta} \gamma^{\mu} q_{L \alpha}, \\
Q_{5} & =\bar{s}_{L \alpha} \gamma_{\mu} b_{L \alpha} \sum_{q=u, ., b} \bar{q}_{R \beta} \gamma^{\mu} q_{R \beta}, \\
Q_{6} & =\bar{s}_{L \alpha} \gamma_{\mu} b_{L \beta} \sum_{q=u, . ., b} \bar{q}_{R \beta} \gamma^{\mu} q_{R \alpha}, \\
Q_{7} & =\frac{e}{16 \pi^{2}} m_{b} \bar{s}_{L} \sigma^{\mu \nu} b_{R} F_{\mu \nu}, \\
Q_{8} & =\frac{g_{s}}{16 \pi^{2}} m_{b} \bar{s}_{L} T^{a} \sigma^{\mu \nu} b_{R} G_{\mu \nu}^{a}, \\
Q_{9} & =\left(\bar{s}_{L} \gamma_{\mu} b_{L}\right) \bar{l} \gamma^{\mu} l, \\
Q_{10} & =\left(\bar{s}_{L} \gamma_{\mu} b_{L}\right) \bar{l} \gamma^{\mu} \gamma_{5} l,
\end{aligned}
$$

$K$ is the CKM-matrix and $q_{L(R)}=\left(1 \mp \gamma_{5}\right) / 2 q$. This Hamiltonian is known at next-to-leading or-

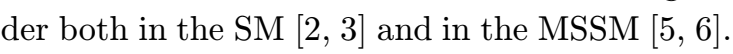
With these definitions the differential branching ratio and the forward-backward asymmetry can be written as

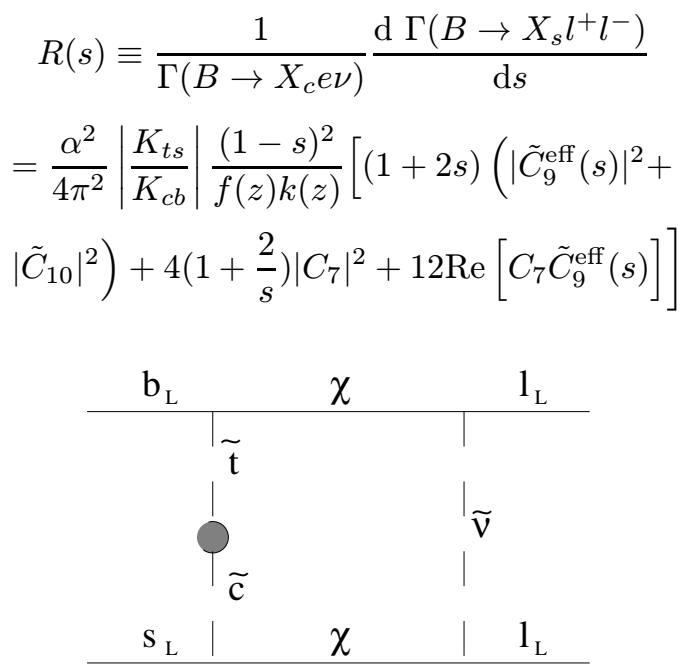

Figure 2: Relevant box diagram for $b \rightarrow s l^{+} l^{-}$. Bubble indicates Mass Insertion. 


$$
\begin{aligned}
A_{F B}(s) & \equiv \frac{\int_{-1}^{1} \mathrm{~d} c \frac{\mathrm{d}^{2} \Gamma}{\mathrm{d} c \mathrm{~d} s} \operatorname{Sgn}(c)}{\int_{-1}^{1} \mathrm{~d} c \frac{\mathrm{d}^{2} \Gamma}{\mathrm{d} c \mathrm{~d} s}} \\
& =-\frac{3 \operatorname{Re}\left[\tilde{C}_{10}\left(s \tilde{C}_{9}^{\text {eff }}(s)+2 C_{7}\right)\right]}{[\cdots]}
\end{aligned}
$$

where $[\cdots]$ is the part of $R(s)$ in square brackets, $\tilde{C}_{i}=\frac{\alpha}{4 \pi} C_{i}, s=\left(p_{l^{+}}+p_{l^{-}}\right)^{2} / m_{b}^{2}, c=\cos \theta$, $\theta$ is the angle between the positively charged lepton and the B flight direction in the rest frame of the dilepton system, $f(z)$ and $k(z)$ are the phase space factor and the QCD correction factor $\left(z=m_{c} / m_{b}\right)$ that enter $\Gamma\left(B \rightarrow X_{c} e \nu\right)$ and can be found in ref. $\left[\begin{array}{lll}\overline{3} & \overline{1} & \overline{1} \\ 2\end{array}\right]$. $\tilde{C}_{9}^{\text {eff }}(s)$ include all the contributions of the operators $Q_{1}-Q_{6}$ and $Q_{8}$ and its complete definition for the SM and

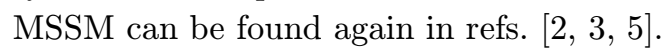

In the literature the energy asymmetry is also considered ["iñ] but it is easy to show that these two kind of asymmetries are completely equivalent; in fact a configuration in the dilepton c.m.s. in which $l^{+}$is scattered in the forward direction kinematically implies $E_{l^{+}}<E_{l^{-}}$in the B rest frame (see for instance ref. [indin).

It is worth underlying that integrating the differential asymmetry given in eq. (2.3i) we do not obtain the global Foward-Backward asymmetry which is by definition:

$$
\frac{N\left(l_{\rightarrow}^{+}\right)-N\left(l_{\leftarrow}^{+}\right)}{N\left(l_{\rightarrow}^{+}\right)+N\left(l_{\leftarrow}^{+}\right)}=\frac{\int_{-1}^{1} \mathrm{~d} c \int \mathrm{d} s \frac{\mathrm{d}^{2} \Gamma}{\mathrm{d} c \mathrm{~d} s} \operatorname{Sgn}(c)}{\int_{-1}^{1} \mathrm{~d} c \int \mathrm{d} s \frac{\mathrm{d}^{2} \Gamma}{\mathrm{d} c \mathrm{~d} s}}
$$

where $l_{\rightarrow}^{+}$and $l_{\leftarrow}^{+}$stand respectevely for leptons scattered in the forward and backward direction. To this extent it is useful to introduce the following quantity

$$
\begin{gathered}
\bar{A}_{F B}(s) \equiv \frac{\int_{-1}^{1} \mathrm{~d} c \frac{\mathrm{d}^{2} \Gamma}{\mathrm{d} c \mathrm{~d} s} \operatorname{Sgn}(c)}{\int_{-1}^{1} \mathrm{~d} c \int \mathrm{d} s \frac{\mathrm{d}^{2} \Gamma}{\mathrm{d} c \mathrm{~d} s}} \\
=\frac{-3 \operatorname{Re}\left[\tilde{C}_{10}\left(s \tilde{C}_{9}^{\mathrm{eff}}(s)+2 C_{7}\right)\right](1-s)^{2}}{\int \mathrm{d} s[\cdots](1-s)^{2}}
\end{gathered}
$$

whose integrated value is given by eq. (2.3i).
All the effects coming from the mass insertion approximation can be included in the previous formulae writing the coefficients $C_{7}, \tilde{C}_{9}^{\text {eff }}(s)$, $\tilde{C}_{10}$ as

$$
\begin{aligned}
C_{7} & =C_{7}^{S M}+C_{7}^{\text {Diag }}+C_{7}^{M I}, \\
\tilde{C}_{9}^{\mathrm{eff}}(s) & =\left(\tilde{C}_{9}^{\mathrm{eff}}(s)\right)^{S M}+\left(\tilde{C}_{9}^{\mathrm{eff}}\right)^{\text {Diag }}+\left(\tilde{C}_{9}^{\mathrm{eff}}\right)^{M I}, \\
\tilde{C}_{10} & =\tilde{C}_{10}^{S M}+\tilde{C}_{10}^{\text {Diag }}+\tilde{C}_{10}^{M I}
\end{aligned}
$$

where all the contributions are evaluated at the $M_{B}$ scale and the various $C_{i}^{D i a g}$ summarize all the contributions coming from graphs including sparticles in the limit in which we neglect all the mass insertion contributions (they would be the only SUSY diagrams if the scalar mass matrices were diagonalized by the same rotations as those needed by the fermions). The explicit expressions for $C_{i}^{\text {Diag }}$ can be found in ref. [isin].

We find that the most general low-energy SUSY lagrangian can also modify (with respect to the SM) the matching coefficients of the operators

$$
\begin{aligned}
Q_{7}^{\prime} & =\frac{e}{8 \pi^{2}} m_{b} \bar{s}_{R} \sigma^{\mu \nu} b_{L} F_{\mu \nu} \\
Q_{9}^{\prime} & =\left(\bar{s}_{R} \gamma_{\mu} b_{R}\right) \bar{l} \gamma^{\mu} l \\
Q_{10}^{\prime} & =\left(\bar{s}_{R} \gamma_{\mu} b_{R}\right) \bar{l} \gamma^{\mu} \gamma_{5} l
\end{aligned}
$$

However the contribution of these operators is negligible and so they are not considered in the final discussion of physical quantities.

Finally the value of the physical constants we use is reported in table 1 .1.

\begin{tabular}{||c|c||}
\hline$m_{t}$ & $173.8 \mathrm{GeV}$ \\
$m_{b}$ & $4.8 \mathrm{GeV}$ \\
$m_{c}$ & $1.3 \mathrm{GeV}$ \\
$m_{s}$ & $125 \mathrm{MeV}$ \\
$M_{B}$ & $5.27 \mathrm{GeV}$ \\
$\alpha_{s}\left(m_{Z}\right)$ & .119 \\
$1 / \alpha_{e l}\left(m_{Z}\right)$ & 128.9 \\
$\sin ^{2} \theta_{W}$ & .2334 \\
\hline
\end{tabular}

Table 1: Central values of physical constants used in the phenomenological analysis

\section{Constraints on mass insertions}

In order to establish how large the SUSY contribution to $B \rightarrow X_{s} l^{+} l^{-}$can be, one can compare, 
coefficient per coefficient, the MI results with the SM ones taking into account possible constraints on the $\delta$ s coming from other processes.

The most relevant $\delta$ s interested in the determination of the Wilson coefficients $C_{7}, C_{9}$ and $C_{10}$ are $\left(\delta_{23}^{u}\right)_{L L},\left(\delta_{23}^{u}\right)_{L R},\left(\delta_{33}^{u}\right)_{R L},\left(\delta_{23}^{d}\right)_{L L}$ and $\left(\delta_{23}^{d}\right)_{L R}$.

- Vaccum stability arguments regarding the absence of color and charge breaking minima and of directions unbounded from below in the potential [i] $\overline{1}_{1}$ give

$$
\left(\delta_{i 3}^{u}\right)_{L R} \leq m_{t} \frac{\sqrt{2 M_{\tilde{u}}^{2}+M_{\tilde{l}}^{2}}}{M_{s q}^{2}} \simeq \sqrt{3} \frac{m_{t}}{M_{s q}} .
$$

For $M_{s q} \leq 300 \mathrm{GeV}$ this is not an effective constraint on the mass insertions.

- A constraint on $\left(\delta_{23}^{d, u}\right)_{L L}$ can come from the possible measure of $\Delta M_{B_{s}}$; in fact the gluino-box contribution to $\Delta M_{B_{s}}$ is proportional to $\left(\delta_{23}^{d}\right)_{L L}^{2}$ (see ref. 1010 ). Assuming

$$
\Delta M_{B_{s}}<30 \mathrm{ps}^{-1}
$$

we find

$$
\left(\delta_{23}^{d}\right)_{L L}<0.5 \text {. }
$$

Moreover the LL $u$ - and $d$ - squark mass matrices are related by a CKM rotation

$$
\left(M_{s q}^{d}\right)_{L L}^{2}=K^{\dagger}\left(M_{s q}^{u}\right)_{L L}^{2} K
$$

so that the limit $(3.3)$ is valid for the up sector too:

$$
\left(\delta_{23}^{u}\right)_{L L}<0.5
$$

- A constraint comes from the measure of $B \rightarrow X_{s} \gamma$. The branching ratio of this process depends almost completely on the Wilson coefficients $C_{7}$ and $C_{7}^{\prime}$ which are dominated by the gluino penguin diagrams involving $\left(\delta_{23}^{d}\right)_{L R}$ or $R L$. The most recent CLEO estimate of the branching ratio for $B \rightarrow X_{s} \gamma$ is [i];]

$$
\mathrm{B}_{\text {exp }}=(3.15 \pm 0.35 \pm 0.32 \pm 0.26) \cdot 10^{-4} \text {. }
$$

where the first error is statistical, the second is systematic and the third comes from the model dependence of the signal. The limits given at $95 \%$ C.L. are [iㅁ]:

$$
2.010^{-4}<\mathrm{B}_{\exp }<4.510^{-4} \text {. }
$$

We can define a $C_{7}^{\text {eff }}\left(M_{B}\right)$ as

$$
\left(C_{7}^{\mathrm{eff}}\left(M_{B}\right)\right)^{2}=\frac{B_{e x p}(B \rightarrow s \gamma)}{\left(\frac{K_{t s} K_{t b}}{K_{c b}}\right)^{2} \frac{6 \alpha F}{\pi g(z)}}
$$

where $F$ can be found in ref. [i] $\left.\overline{3}_{1}\right]$. Considering the limits in eq. $\left(\begin{array}{c}\overline{3} \cdot \overline{7} \\ \mathbf{3}\end{array}\right)$ we find

$$
0.250<\left|C_{7}^{\text {eff }}\left(M_{B}\right)\right|<0.445 .
$$

In a general SUSY theory $\left|C_{7}^{\text {eff }}\left(M_{B}\right)\right|^{2}=$ $\left|C_{7}\left(M_{B}\right)\right|^{2}+\left|C_{7}^{\prime}\left(M_{B}\right)\right|^{2}$ and the constraint given in eq. (3.9i) should be shared between the two coefficients. However in order to get the maximum SUSY contribution, we observe that in physical observables we are interested in $C_{7}^{\prime}$ does not interfere with $C_{7}$, the $C_{7}^{\prime} C_{9}$ term is suppressed by a factor $m_{s} / m_{b}$ with respect to the $C_{7} C_{9}$ one and $C_{7}^{\prime} C_{9}^{\prime}$ is numerically negligible (in fact $C_{9}^{\prime}$ is much smaller than $C_{9}$ ). For these reasons we choose to fill the constraint of eq. (3.9.) with $C_{7}\left(M_{B}\right)$ alone (note that $\left(\delta_{23}^{d}\right)_{L R}$ and $\left(\delta_{23}^{d}\right)_{R L}$ are independent parameters).

The bounds $\left(\mathbf{3}^{-} . \overline{9}\right)$ are referred to the coefficient evaluated at the $M_{B}$ scale while we are interested to the limits at the much higher matching scale. After the RG evolution has been performed we find

$$
\left\{\begin{array}{l}
-0.39<C_{7}\left(M_{W}\right)<-0.099 \\
0.66<C_{7}\left(M_{W}\right)<0.95
\end{array}\right.
$$

For $M_{s q}$ lower than $1000 \mathrm{GeV}$, the MIA contribution alone with a suitable choice of $\delta \mathrm{s}$, can always fit the experimental constraints.

Thus, since we are interested in computing the maximum enhancement (suppression) SUSY can provide, we can choose the total $C_{7}^{e f f}\left(M_{B}\right)$ anywhere inside the allowed region given in eq. (3.9.) still remaining consistent with the MIA.

The limit we get for $\left(\delta_{23}^{d}\right)_{L R}$ from eq. ( $\left.\overline{3} \cdot \overline{1} \overline{0_{1}}\right)$ is of order $10^{-2}$ and this rules out gluino $\mathrm{Z}_{-}^{-}$ penguins contributions to $C_{9}$ and $C_{10}$. 
- Finally a comment on the $\delta$ s coming in graphs with a double MI is in order.

Given the constraints on $\left(\delta_{23}^{d}\right)_{R L}$ one can see that the gluino-penguins with a double MI give negligible contributions to the final results even if $\left(\delta_{33}^{d}\right)_{R L}$ is of order $\mathcal{O}(1)$.

A $\left(\delta_{33}^{u}\right)_{R L}$ of order $\mathcal{O}(1)$, can give rise to light or negative squark mass eigenstates. In any case the numerical value of these contributions is not particularly important for the determination of physical observables. Since we want to provide a model independent analysis we prefer not to consider in our final computation these double insertion graphs.

\section{Results}

The results of the calculations of the Wilson coefficients are presented in figs. we sum all contributions coming from different graphs proportional to a common mass insertion (the actual values of the coefficients are obtained multiplying the points in the plots by the MI).

With $\mu \simeq-160, M_{g l} \simeq M_{s q} \simeq 250 \mathrm{GeV}$, $M_{\tilde{t}_{R}} \simeq 70 \mathrm{GeV}, M_{\tilde{\nu}} \simeq 50 \mathrm{GeV}, \tan \beta \simeq 2$ one gets

$$
\left\{\begin{aligned}
C_{9}^{M I}\left(M_{B}\right)=-1.2\left(\delta_{23}^{u}\right)_{L L}+0.69\left(\delta_{23}^{u}\right)_{L R^{-}} & 0.51\left(\delta_{23}^{d}\right)_{L L} \\
C_{10}^{M I}\left(M_{B}\right)= & 1.75\left(\delta_{23}^{u}\right)_{L L}-8.25\left(\delta_{23}^{u}\right)_{L R}
\end{aligned}\right.
$$

According to the discussion of section we find that, for diagrams involving charginos, the various contributions enhance the $\mathrm{SM}$ prediction in the most efficient way choosing $\left(\delta_{23}^{u}\right)_{L R}=0.9$, $\left(\delta_{23}^{u}\right)_{L R}=-0.5 . C_{9}$ receives a very small contribution also from gluino penguins.

The extremal values of $C_{9}$ and $C_{10}$, obtained with the above choice of $\delta$ s are:

$$
\left\{\begin{array}{l}
C_{9}^{M I}\left(M_{B}\right)= \pm 1.5 \\
C_{10}^{M I}\left(M_{B}\right)=\mp 8.4
\end{array}\right.
$$

The relative sign between $C_{9}^{M I}$ and $C_{10}^{M I}$ is fixed, as they are proportional to the same $\delta$ s.

In order to numerically compare eq. (4.2) with the respective SM results we note that the minimum value of $\left(C_{9}^{\text {eff }}(s)\right)^{S M}\left(M_{B}\right)$ is about 4.3 while $C_{10}^{S M}=-4.6$. Thus, from eq. (4.2i) one deduces that SM expectations for the observables are enhanced when $C_{9}^{M I}\left(M_{b}\right)$ is positive. Moreover the big value of $C_{10}^{M I}\left(M_{B}\right)$ implies that the final total coefficient $C_{10}\left(M_{B}\right)$ can have a different sign with respect to the SM estimate. As a consequence of this, the sign of asymmetries can be the opposite of the one calculated in the SM.

The diagonal contributions to $C_{9}, C_{10}$ introduced in sec.5. of the parameters are

$$
\left\{\begin{array}{l}
C_{9}^{\text {diag }}\left(M_{B}\right)=-0.35 \\
C_{10}^{\text {diag }}\left(M_{B}\right)=-0.27
\end{array}\right.
$$

The sign and the value of the coefficient $C_{7}$ has a great importance too. In fact the integral of the BR is dominated by the $\left|C_{7}\right|^{2} / s$ and $C_{7} C_{9}$ term for low values of $s$. In the SM the interference between $O_{7}$ and $O_{9}$ is destructive and this behavior can be easily modifed in the general class of models we are dealing with.

We find the maximum enhancement of the BR and $A_{F B}$ with $C_{7}^{\text {eff }}=0.445$ and of $\bar{A}_{F B}$ with $C_{7}^{\text {eff }}=0.250$ while $C_{9}^{M I}=1.5$ and $C_{10}^{M I}=-8.4$. It is important to note that with such choices the behavior of the asymmetries in the low s region of the spectrum is greatly modified: the coefficients of the operators $Q_{7}$ and $Q_{9}$ sum up instead of cancel each other in such a way that the asymmetries are never negative.

We compute, in addition, the best enhancement compatible with the condition of keeping the SM sign of $C_{7}^{e f f}$, that is with $C_{7}^{\text {eff }}=-0.445$ for the BR and $C_{7}^{\text {eff }}=-0.250$ for asymmetries with the same values of $C_{9}^{M I}$ and $C_{10}^{M I}$.

The most negative value for the asymmetries corresponds to $C_{7}^{\text {eff }}=-0.250, C_{9}^{M I}=-1.5$ and $C_{10}^{M I}=8.2$. The strongest suppression of the SM result for the branching ratio is instead obtained for $C_{7}^{e f f}=-0.445$ and with $\delta$ s such that $C_{10}^{M I}$ complitely annihilates $C_{10}^{S M}$. This implies $\left(\delta_{23}^{u}\right)_{L R}=-0.5$ and $\left(\delta_{23}^{u}\right)_{L L}=0.5$ in eq. (4.1.i. $)$ so that $C_{9}^{M I}=-1.5$.

The plots of $B R(s), A_{F B}(s)$ and $\bar{A}_{F B}(s)$ are drawn in figs. $\bar{p}_{1}^{1} \overline{6}$. Here both SM and SUSY results are shown. The discontinuity in the $A_{F B}$ plot at $s=0.7$ corresponds to the point at which we have stopped the corrections $O\left(1 / m_{b}^{2}\right)$. In 

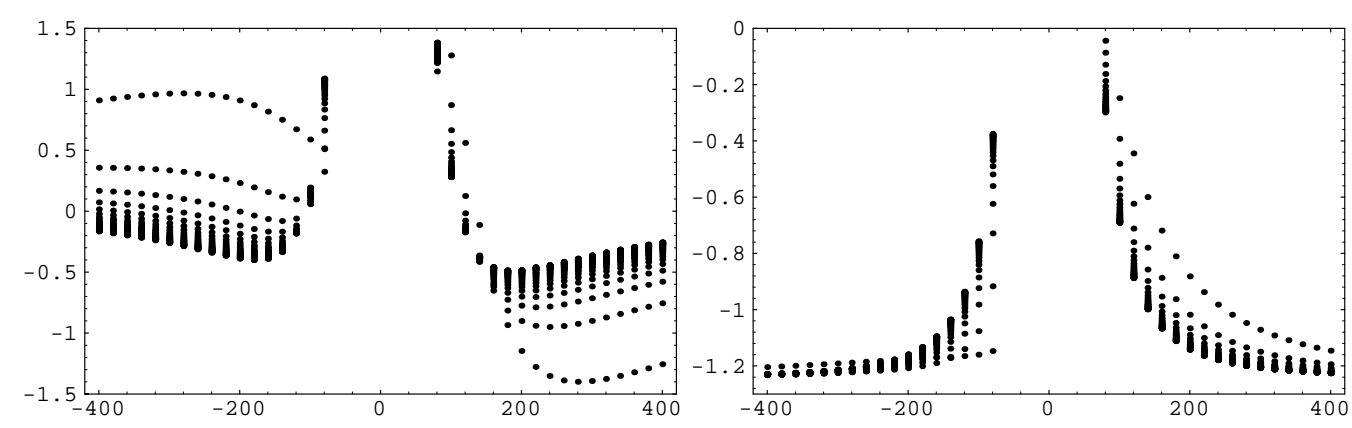

Figure 3: $\left(\delta_{23}^{u}\right)_{L R}$ (left) and $\left(\delta_{23}^{u}\right)_{L L}$ (right) contributions to $C_{9}$ coming from chargino diagrams as functions of $\mu$ (expressed in $\mathrm{GeV}$ ). $M_{s q}$ is fixed to $250 \mathrm{GeV}$ while $\tan \beta$ varies between 2 and 50.
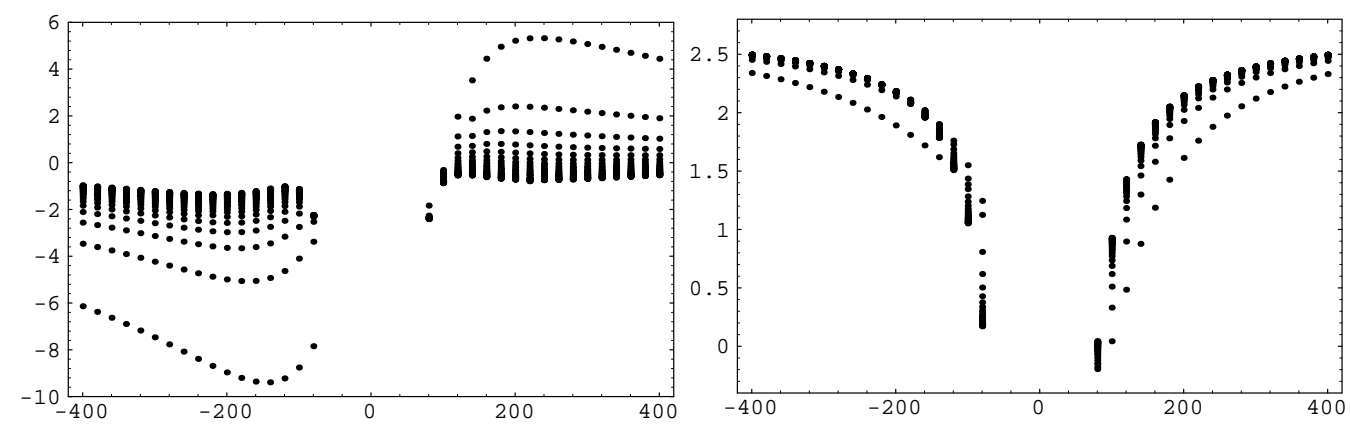

Figure 4: $\left(\delta_{23}^{u}\right)_{L R}$ (left) and $\left(\delta_{23}^{u}\right)_{L L}$ (right) contributions to $C_{10}$ coming from chargino diagrams as functions of $\mu$ (expressed in $\mathrm{GeV}$ ). $M_{s q}$ is fixed to $250 \mathrm{GeV}$ while $\tan \beta$ varies between 2 and 50.

fact a model independent description of the differential asymmetry in the region $0.7<s<0.93$ beyond the parton model one still lacks. Further the peak wich occur at $s=\left(2 m_{c} / m_{b}\right)^{2} \simeq 0.3$ is due to the perturbative remnant of the $c \bar{c}$ resonance.

The integrated BRs and asymmetries for the decays $B \rightarrow X_{s} e^{+} e^{-}$and $B \rightarrow X_{s} \mu^{+} \mu^{-}$in the SM case and in the SUSY one (with the above choices of the parameters) are summarized in tab. 2. There we computed the total perturbative contributions without the resonances; these occur in the intermediate range of the spectrum $\left(J / \psi\right.$ at $3.1 \mathrm{GeV}(s=0.42)$ and $\psi^{\prime}$ at $3.7 \mathrm{GeV}$ $(s=0.59)$ plus others at higher energies). However it is possible to exclude the resonant re- gions from the experimental analysis by opportune cuts and to correct the effects of their tails in the remaining part of the spectrum.

The results of tab. ${ }_{2}^{2}$ must be compared with the experimental best limit which reads [1]

$$
B R_{e x p}<5.810^{-5}
$$

A comment on the Constrained MSSM prediction for the observables we have computed is now necessary. An analysis on the subject is presented in ref. [5] that the effect of CMSSM on the integrated BRs, considering only contributions to $C_{9}$ and $C_{10}$, varies between a depression up to $10 \%$ and an enhancement of few percents relative to the corresponding SM values. The asymmetries get even 


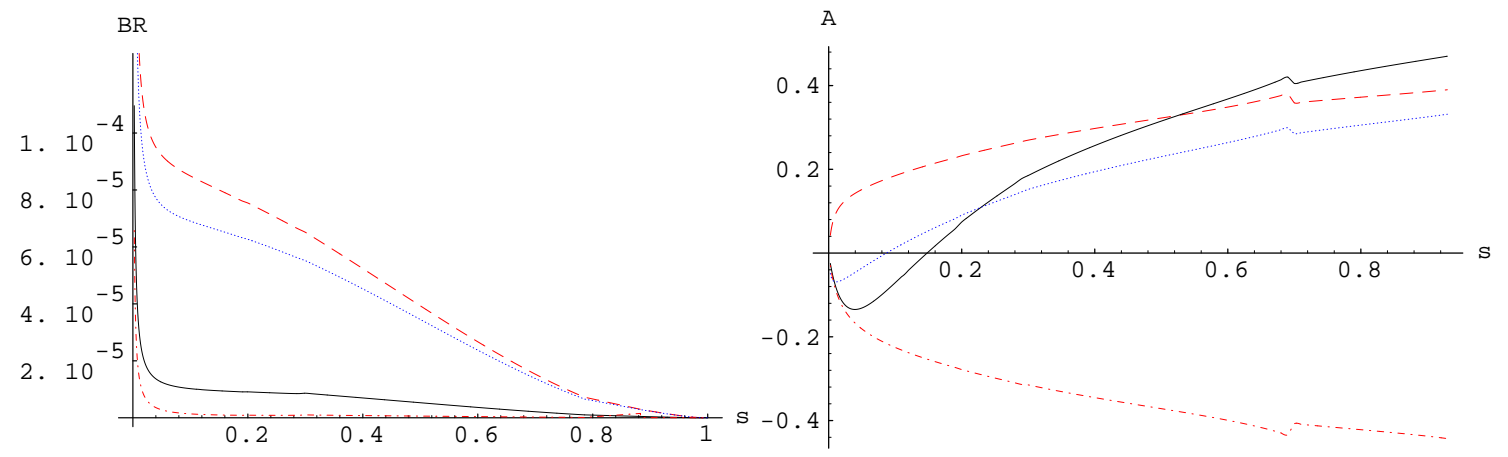

Figure 5: Differential branching ratio [left] and forward-backward asymmetry ( $\left.A_{F B}\right)$ [right] for the decay $B \rightarrow X_{s} l^{+} l^{-}$. The solid line corresponds to the SM expectation; the dashed and dotted-dashed lines correspond respectively to the SUSY best enhancement and depression; the dotted line is the maximum enhancement obtained without changing the sign of $C_{7}$.

\begin{tabular}{||c|c|c|c||}
\hline & SM & $\begin{array}{c}\text { SUSY } \\
\text { maximal }\end{array}$ & $\begin{array}{c}\text { SUSY } \\
\text { minimal }\end{array}$ \\
\hline \hline$B R(e)$ & $8.610^{-6}$ & $4.810^{-5}$ & $2.810^{-6}$ \\
\hline$A_{F B}(e)$ & 0.23 & 0.28 & -0.32 \\
\hline $\bar{A}_{F B}(e)$ & 0.077 & 0.21 & -0.21 \\
\hline \hline$B R(\mu)$ & $5.810^{-6}$ & $4.210^{-5}$ & $1.010^{-6}$ \\
\hline$A_{F B}(\mu)$ & 0.23 & 0.28 & -0.32 \\
\hline $\bar{A}_{F B}(\mu)$ & 0.11 & 0.22 & -0.28 \\
\hline
\end{tabular}

Table 2: Integrated $B R, A_{F B}$ and $\bar{A}_{F B}$ in the $S M$ and in a general SUSY extension of the SM for the decays $B \rightarrow X_{s} e^{+} e^{-}$and $B \rightarrow X_{s} \mu^{+} \mu^{-}$. The second and third columns are the extremal values we obtain with a positive $C_{7}^{\text {eff }}$ while the fourth one is the $C_{7}^{e f f}<0$ case. The actual numerical inputs for the various coefficients can be found in the text. The BR is just the integral of $R(s)$ multiplied by the $B R$ of the semileptonic dominant $B$ decay $\left(B R\left(B \rightarrow X_{c} e \nu\right)=\right.$ $0.105)$.

smaller corrections. On the other hand a direct

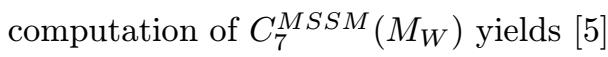

$-0.59<C_{7}^{M S S M}\left(M_{W}\right)<+0.49 \quad$ large $\tan \beta$,

$-0.26<C_{7}^{M S S M}\left(M_{W}\right)<-0.20 \quad$ low $\tan \beta$.

It is worth observing that the limits given in

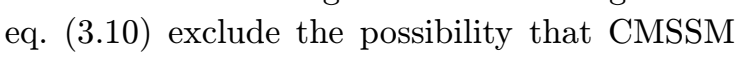

could drive a positive value for $C_{7}^{e f f}\left(M_{B}\right)$. For what concerns the negative interval of values of $C_{7}^{e f f}\left(M_{B}\right)$ we see that it can be accommodated both in the CMSSM and in our framework.

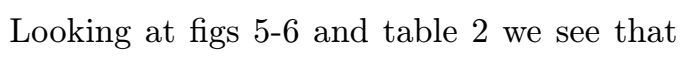
the differences between SM and SUSY predictions can be remarkable. Moreover a sufficiently precise measure of BRs, $A_{F B} \mathrm{~S}$ and $\bar{A}_{F B} \mathrm{~S}$ can either discriminate between the CMSSM and more general SUSY models or give new constraints on mass insertions. Both these kind of informations can be very useful for model building.

\section{Acknowledgments}

We thank S. Bertolini and E. Nardi for fruitful discussions. I.S. wants to thank SISSA, for support and kind hospitality during the elaboration of the first part of this work and Della Riccia Foundation for partial support. This work is partially supported by INFN and by the TMR-EEC network "Beyond the Standard Model" (contract number ERBFMRX CT960090).

\section{References}

[1] See, for instance, The BaBar Physics Book, SLAC-R-504 and references therein.

[2] G. Buchalla and A. J. Buras, Nucl. Phys. B400 (1993), 225; 

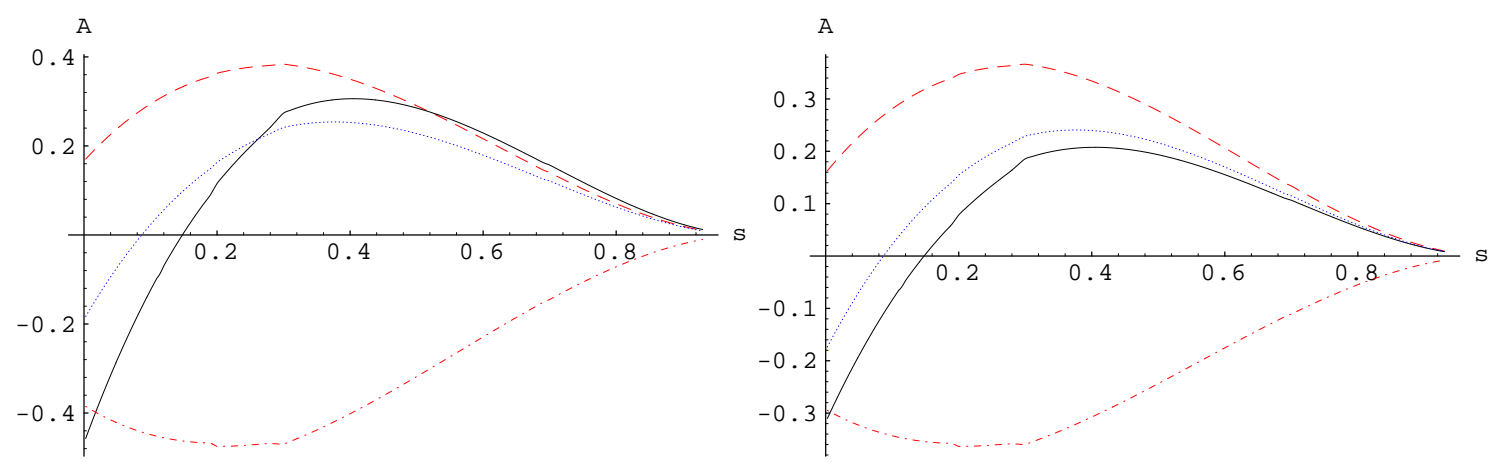

Figure 6: Forward-Backward asymmetry $\left(\bar{A}_{F B}\right)$ for the decays $B \rightarrow X_{s} \mu^{+} \mu^{-}$[left] and $B \rightarrow X_{s} e^{+} e^{-}$[right] The solid line corresponds to the SM expectation; the dashed and dotted-dashed lines correspond respectively to the SUSY best enhancement and depression; the dotted line is the maximum enhancement obtained without changing the sign of $C_{7}$.

M. Misiak, Nucl. Phys. B383 (1993) 23, erratum ibid. B439 (1995) 461;

A.J. Buras and M. Münz, Phys. Rev. D52 (1995) 186.

[3] G. Buchalla, A.J. Buras and M.E. Lautenbacher, Rev. Mod. Phys. 68 (1996) 1125.

[4] A. Ali, G. Hiller, L. T. Handoko and T. Morozumi, Phys. Rev. D55 (1997) 4105;

A. Ali and G. Hiller, Phys. Rev D58 (1998) 071501 and Phys. Rev D58 (1998) 074001.

[5] P. Cho, M. Misiak and D. Wyler, Phys. Rev. D54 (1996) 3329

[6] T. Goto, Y. Okada, Y. Shimizu and M. Tanaka, Phys. Rev. D55 (1997) 4273-4289.

[7] CLEO Collaboration, CONF98-17, ICHEP98, 1011.

[8] L. J. Hall, V. A. Kostolecki and S. Raby, Nucl. Phys. B267 (1986) 415.

[9] F. Gabbiani and A. Masiero, Nucl. Phys. B322 (1989) 235.

[10] F. Gabbiani, E. Gabrielli, A. Masiero and L. Silvestrini, Nucl. Phys. B477 (1996) 321.

[11] J.A. Casas and S. Dimopoulos, Phys. Lett. B387 (1996) 107.

[12] N. Cabibbo and L. Maiani, Phys. Lett. B79 (1978) 109;

C.S. Kim and A.D. Martin, Phys. Lett. B225 (1989) 186.
[13] K. Chetyrkin, M. Misiak and M. Münz, Phys. Lett. B400 (1997) 206-219;

Erratum-ibid. B425 (1998) 414.

[14] S. Glenn et al. (CLEO Collaboration), Phys. Rev. Lett. 80 (1998) 2289. 